\title{
Ternary QD-Organic Dye FRET Pairs for Cytometry-Compatible Sensing Platform
}

\author{
Maksim Miropoltsev $^{1}$, Vera Kuznetsova ${ }^{1}$, Viktoria Osipova ${ }^{1}$, Anton Tkach ${ }^{1}$, Alexander Baranov ${ }^{1}$ \\ ${ }^{1}$ Center of Information Optical Technology, ITMO University \\ 197101 Saint Petersburg, Russia \\ miropoltsev_m@niuitmo.ru; v.kuznetsova.a@gmail.com; vaosipova@itmo.ru; aptkach@itmo.ru; a_v_baranov@itmo.ru
}

\section{Extended Abstract}

Ternary quantum dots (QDs), such as $\operatorname{AgInS}_{2}$ (AIS), are very promising nanomaterials with a range of potential applications in photovoltaics and biomedicine [1]. Low toxicity, high absorption coefficients, inherently broadband photoluminescence (PL), large Stokes shifts, and long wavelength-dependent lifetimes have made these nanoparticles the objects of extensive research in the last decades [2]. Due to unique photophysical properties, it can be advantageous to use ternary QDs as energy donors in the systems with Förster resonance energy transfer (FRET) to build high-performance sensing platforms and multiplex assays with both spectral and lifetime encoding [3]. Nevertheless, there is still the lack of information regarding the mechanisms of ternary QD photoluminescence and the suitability of ternary QDs for the FRET-based applications.

In the present study, we have prepared ternary QD-organic dye FRET pairs using the double-shell AIS/ZAIS/ZnS QDs and $\mathrm{Cy} 3$ or $\mathrm{Cy} 5$ cyanine dyes by the means of an electrostatic interaction in aqueous solutions. We have investigated the recombination pathways of the free QDs and the QD-dye complexes using time-resolved fluorescence spectroscopy. Finally, we have incorporated similar FRET pairs into the polymer microspheres (PMS) via layer-by-layer deposition technique to fabricate the flow cytometry-compatible fluorescent labels.

At the first stage, we investigated the temporal characteristics of the AIS/ZAIS/ZnS QD photoluminescence. We used the three-exponential model to fit the PL decay curves of the free nanoparticles. For the complex formation, we chose the cyanine dyes with the absorbance overlapping with the quantum dot PL in different spectral regions, that allowed us to achieve selective quenching of the QD emission. Our studies show that FRET results in a decrease of all QD PL decay components. It was found that the shortest lifetime decreases the most, while the longest one decreases the least. The obtained results suggest that both the radiative recombination rate and the defect location can alter the FRET efficiency of a particular recombination channel. Our data is consistent with the model stating that the shortest component corresponds to the surface defects, and the longest - to the QD core. We have also analyzed the sensitized acceptor emission and conclude that the transferred energy is primarily coming from the QD alloy layer with the high concentration of defects.

At the second stage, we prepared polymer microspheres encoded with AIS/ZnS QDs and Cy3 of Cy5 cyanine dyes via layer-by-layer deposition using positive and negative polyelectrolytes as separators. Our studies show that the sensitized dye PL intensity demonstrates a clear maximum in case of two double polyelectrolyte layers between the QDs and the dye molecules. Time-resolved measurements indicate that the PL lifetime decreases for the QDs and increases for the dyes because of FRET. The results clearly demonstrate the potential to record the spectrally resolved bands of FRET-induced luminescence for dyes with different decay times.

To conclude, we believe that our studies provide substantial contribution towards better understanding of the ternary QD emission mechanisms, as well as possible ways of utilizing ternary QDs as energy donors in the systems with FRET. The proposed PMS-QD-dye model can be implemented, for instance, to produce flow cytometry-compatible sensing platforms and multiplex assays with both spectral and lifetime encoding.

This research was funded by Federal Target Program for Research and Development of the Ministry of Science and Higher Education of the Russian Federation, grant number 14.587.21.0047, identifier RFMEFI58718X0047. 


\section{References}

[1] Girma, W.M., Fahmi, M.Z., Permadi, A., Abate, M.A. and Chang, J.Y., 2017. Synthetic strategies and biomedical applications of I-III-VI ternary quantum dots. Journal of Materials Chemistry B, 5(31), pp.6193-6216.

[2] Moodelly, D., Kowalik, P., Bujak, P., Pron, A. and Reiss, P., 2019. Synthesis, photophysical properties and surface chemistry of chalcopyrite-type semiconductor nanocrystals. Journal of Materials Chemistry C, 7(38), pp.11665-11709.

[3] Kuznetsova, V., Tkach, A., Cherevkov, S., Sokolova, A., Gromova, Y., Osipova, V., Baranov, M., Ugolkov, V., Fedorov, A. and Baranov, A., 2020. Spectral-Time Multiplexing in FRET Complexes of AgInS2/ZnS Quantum Dot and Organic Dyes. Nanomaterials, 10(8), p.1569. 